Check for updates

Cite this: RSC Adv., 2018, 8, 33473

\title{
Transition metal-promoted hierarchical ETS-10 solid base for glycerol transesterification
}

\begin{abstract}
Mei Xiang (iD) and Dongfang $\mathrm{Wu}^{*}$
A transesterification reaction has been carried out over a transition metal modified hierarchical ETS-10 (METS-10) catalyst to synthesize glycerol carbonate (GC) from glycerol. The inherent Lewis basicity of ETS-10 favors oriented conversion of glycerol, and the hierarchical structure benefits exposure of more active sites, shortens the molecular diffusion path, suppresses the formation of coke in the micropores, and then enhances the catalytic reactivity and stability. Furthermore, the influence of transition metals ( $\mathrm{Fe}, \mathrm{Co}, \mathrm{Ni}, \mathrm{Cu}, \mathrm{Zn}$ and $\mathrm{Mn}$ ) on the basic sites of supports has been investigated in detail. It is found that basicity change of the catalyst depends on not only the cation size, nature and composition of the transition metal but also the zeolite structure and pore topology. Besides, the presence of a certain amount of $\mathrm{Ni}^{0}$ species from catalyst reduction has proved to play a critical role in strengthening the interaction of Lewis basic sites $\left(\mathrm{TiO}_{6}{ }^{2-}\right.$ ) with active glycerol hydroxyl groups. Finally, a $97.7 \%$ glycerol conversion and $97.1 \%$ GC yield have been obtained over Ni/METS-10, of which the high catalytic performance can be maintained after 8 runs.
\end{abstract}

Received 14th August 2018

Accepted 22nd September 2018

DOI: 10.1039/c8ra06811a

rsc.li/rsc-advances

\section{Introduction}

The surplus of glycerol from the booming biodiesel industry (about $10 \mathrm{wt} \%$ of the total production) has attracted widespread attention to take full advantage of its application potential. ${ }^{1-3}$ There have been much research focusing on the transformation of glycerol into value-added chemicals, among which glycerol carbonate (GC) is one of the most promising derivatives, characterized by low toxicity, corrosiveness, volatility and flammability, high boiling point, strong polarity, excellent water solubility and biodegradability. ${ }^{4-6}$ As a result, GC has been made wide use of in the food, cosmetics, textile, pharmaceuticals, manufacturing, new energy and materials industries. ${ }^{7-9}$ Various synthetic pathways have been reported for GC preparation from glycerol and different sources of carbonyl functionality such as carbon dioxide, carbon monoxide, phosgene and urea. ${ }^{10-14}$ It was once thought to be the most ideal route to directly carbonate glycerol with carbon dioxide. However, taking the poor production yield and harsh reaction conditions into consideration, it will definitively suffer from nonnegligible production costs and commercialization limitation. Another method for GC synthesis is to use urea as carboxylating agent with distinct advantages of low starting material cost and relatively high GC yield, ${ }^{5,7}$ while the requirement of continuously removing the evolved ammonia gas to accelerate the reaction rate significantly hinders its further applications. In addition, the two undesirable side products

Department of Chemical Engineering, School of Chemistry and Chemical Engineering, Southeast University, Jiangning District, Nanjing 211189, China. E-mail: dfwu@seu. edu.cn isocyanic acid and biuret are troublesome. Also, using dimethyl carbonate (DMC) or diethyl carbonate (DEC) to synthesis GC via transesterification with glycerol is a extensively studied route, and has proved to be much safer and greener due to the low toxicity of raw material, mild operation condition, high selectivity and easy purification of GC. ${ }^{15-20}$ Hence, among these well-known methods, the transesterification of glycerol with DMC/DEC for producing GC is the most promising and suitable industrial process, which has been gained more and more research attention with a large quantity of homogeneous and heterogeneous base catalysts being investigated.

For transesterification reaction of glycerol, typically homogeneous base catalysts such as the alkali metal compounds $\left(\mathrm{KOH}, \mathrm{K}_{2} \mathrm{CO}_{3}\right.$ and $\mathrm{NaOH}$ ) have been reported with favorable performance and some even obtained nearly complete glycerol conversion, but the inconvenience of catalysts separation from reaction system cannot be solved effectively. ${ }^{21,22}$ Accordingly, heterogeneous catalysts seem to be more competitive and desirable. Among them, alkaline earth metal oxides especially $\mathrm{CaO}$ and $\mathrm{MgO}$, and hydrotalcites are the most frequently used catalysts, which all prove to be high active. ${ }^{23-26}$ Others such as $\mathrm{Mg} / \mathrm{Al} / \mathrm{Zr}$ mixed oxide, $\mathrm{Co}_{3} \mathrm{O}_{4} / \mathrm{ZnO}, \mathrm{Mg}-\mathrm{Ca}, \mathrm{Mg}-\mathrm{Li}, \mathrm{LiNO}_{3} /$ $\mathrm{Mg}_{4} \mathrm{AlO}_{55}, \mathrm{Li} / \mathrm{ZnO}$ and ion liquids also have been explored to efficiently transform glycerol into GC. ${ }^{27-31}$ Whereas, many of these catalysts are still subjected to poor reusability, long reaction time, high energy consumption and demand for more than one solvents. Therefore, developing highly stable, reusable and efficient heterogeneous catalysts for the selective synthesis of GC under mild, economical and environment-friendly conditions is of great significance. 
We have reported earlier a heterogeneous ETS-10 catalyst with intrinsic and strong Lewis basic property, which presented a remarkably catalytic performance for the preparation of $\alpha, \beta$ unsaturated carbonyl compounds. ${ }^{32}$ Actually, ETS-10, a titanosilicate molecular sieve with a 3D 12-ring structure, has attracted considerable attention owing to its distinguished potential in adsorption, ion exchange and shape-selective catalysis. ${ }^{33,34}$ Moreover, its unique framework that comprises corner sharing $\mathrm{TiO}_{6}{ }^{2-}$ octahedra and $\mathrm{SiO}_{4}$ tetrahedra as building units gives rise peculiar properties, especially the octahedrally coordinated titanium(Iv) carrying two negative charges endows ETS-10 with characteristic basicity. ${ }^{35}$ Hence, ETS-10, as a prospective heterogeneous base catalyst, can be envisioned to make an indispensable contribution for glycerol transesterification.

Recently, the promoting effect of transition metal in enhancing surface basic property of hydrotalcites have been detailedly discussed and have successfully applied in GC production from transesterification reaction by Liu et al. ${ }^{36}$ While for ETS-10, only Cs have been widely employed to investigate its influence on the alterations of basicity, ${ }^{37}$ and there have not been in-depth and systematic research regarding the effects of impregnating transition metals on the basicity of ETS10 , as well as the catalytic performance. Besides, ETS-10 with hierarchically porous structure has not yet been studied in GC synthesis. Herein, a series of ETS-10 catalysts were prepared and impregnated with transition metals ( $\mathrm{Fe}, \mathrm{Co}, \mathrm{Ni}, \mathrm{Cu}, \mathrm{Zn}$ and $\mathrm{Mn}$ ) for glycerol transesterification with DMC. The structure and catalytic activity correlations of various ETS-10 catalysts were discussed in detail. Furthermore, the kinetic investigation and stability test were also conducted from the aspect of different reaction conditions including reaction temperature and time, the molar ratio of DMC to glycerol, the mass of catalyst and metal loading.

\section{Experimental methods}

\subsection{Chemicals}

Glycerol (ACS, $\geq 99.5 \%$ ) were purchased from Macklin Biochemical Co., Ltd (Shanghai, China). Iron nitrate nonahydrate, cobalt nitrate hexahydrate, nickel nitrate hexahydrate, cupric nitrate trihydrate, zinc nitrate hexahydrate and 50\% manganous nitrate solution, dimethyl carbonate (DMC), diethyl carbonate (DEC), glycerol carbonate (GC), $n$-butanol, benzyl alcohol were all analytical reagent grade and obtained from Sinopharm Chemical Reagent Co., Ltd. Others were also of analytical grade and used as-received without further purification.

\subsection{Catalyst preparation}

ETS-10 was prepared by a molar composition of $1.0 \mathrm{TiO}_{2} /$ $5.5 \mathrm{SiO}_{2} / 3.5 \mathrm{Na}_{2} \mathrm{O} / 1.6 \mathrm{~K}_{2} \mathrm{O} / 181.0 \mathrm{H}_{2} \mathrm{O}$. In a typical run, $16.6 \mathrm{~mL}$ aqueous waterglass solution $\left(\mathrm{SiO}_{2} / \mathrm{Na}_{2} \mathrm{O}\right.$ molar ratio: 3.55, $\rho$ : $1.32 \mathrm{~g} \mathrm{~cm}^{-3}$ ) was mixed with $32.8 \mathrm{~mL} \mathrm{H}_{2} \mathrm{O}$ after stirred at room temperature for about $0.3 \mathrm{~h}$. Subsequently, $6.8 \mathrm{~g} \mathrm{NaCl}, 1.39 \mathrm{~g}$ $\mathrm{KOH}$ and $1.39 \mathrm{~g} \mathrm{KF}$ were added under vigorous stirring. After further addition of $1.3 \mathrm{~g}$ P25 and stirring for $2 \mathrm{~h}$, the obtained gel was transferred into a Teflon-coated stainless-steel autoclave for crystallization at $230{ }^{\circ} \mathrm{C}$ for $72 \mathrm{~h}$. The resulting product was filtered, washed, dried at $100^{\circ} \mathrm{C}$ overnight before calcined in air at $475^{\circ} \mathrm{C}$ for $5 \mathrm{~h}$. The hierarchical porous ETS-10 (METS-10) was synthesized in the same way with an additional role of cationic copolymer COPQA (COPQA $/ \mathrm{TiO}_{2}$ molar ratio: 0.0068). $\mathrm{M}_{1}$ ETS$10, \mathrm{M}_{2}$ ETS-10 and $\mathrm{M}_{3}$ ETS-10 were obtained by adding different amount of COPQA. To prepare transition metal loaded samples, METS-10 was incipient-wetness impregnated with corresponding metal nitrate $\left(m_{\text {metal }} / m_{\text {METS-10 }}=0.05\right)$ and then calcined at $475{ }^{\circ} \mathrm{C}$ after drying treatment.

\subsection{Catalyst characterization}

Powder X-ray diffraction patterns (XRD) were obtained with a Rigaku Ultima IV powder X-ray diffractometer using $\mathrm{CuK} \alpha$ radiation $(\lambda=0.1542 \mathrm{~nm})$. The scan range is from $5^{\circ}$ to $45^{\circ}$. Agilent ICPOES-730 inductive coupled plasma-atomic emission spectrometer (ICP-AES) was employed for elemental analyses. Nitrogen physisorption was conducted at $-196{ }^{\circ} \mathrm{C}$ on a Micromeritics ASAP 2020M apparatus. The sample was degassed for $8 \mathrm{~h}$ at $300{ }^{\circ} \mathrm{C}$ before measurement. Specific surface area was calculated from the adsorption data using the BrunauerEmmett-Teller (BET) equation. The pore size distribution was calculated according to the Barrett-Joyner-Halenda (BJH) model using adsorption data. Scanning electron microscopy (SEM) was performed using a FEI Inspect F50. Transmission electron microscope (TEM) images were collected using a FEI Tech 20 field emission electron microscope with a limited line resolution capacity of $0.14 \mathrm{~nm}$, under a voltage of $200 \mathrm{kV}$. Before characterization by the TEM technique, the sample was cut into thin slices and dropped onto a Cu-grid coated with carbon membrane.

The catalysts basicity was tested by temperatureprogrammed desorption of carbon dioxide $\left(\mathrm{CO}_{2}\right.$-TPD) on a Micromeritics ASAP2920 instrument. A $200 \mathrm{mg}$ sample placed in a quartz tube was pretreated in hydrogen with a flow of 150 $\mathrm{ml} \min ^{-1}$ at $400{ }^{\circ} \mathrm{C}$ for $2 \mathrm{~h}$, and then was activated in helium at this temperature for an hour before cooled to $50^{\circ} \mathrm{C}$. After pulse adsorption of $\mathrm{CO}_{2}$ for $3 \mathrm{~h}$ and being purged with helium flow for $1 \mathrm{~h}$, the spectrum was recorded from $50^{\circ} \mathrm{C}$ to $700^{\circ} \mathrm{C}$ at a heating rate of $10^{\circ} \mathrm{C} \mathrm{min}^{-1}$. CO chemisorption was also measured on the same instrument with a necessary preprocess for the catalyst sample in $\mathrm{H}_{2}$ at $400{ }^{\circ} \mathrm{C}$ for $2 \mathrm{~h}$. After purged with He flow for $60 \mathrm{~min}$ and cooled to room temperature, the sample was exposed to10 vol\% CO-He pulse until CO adsorption saturation. The $\mathrm{CO}$ uptake was measured by a TCD detector. For $\mathrm{H}_{2}$-TPR experiments, the samples were firstly activated with nitrogen flow for $2 \mathrm{~h}$ at $400{ }^{\circ} \mathrm{C}$ and analyzed in a $10 \% \mathrm{H}_{2} / \mathrm{Ar}$ gas mixture $\left(30 \mathrm{~cm}^{3} \mathrm{~min}^{-1}\right)$ from room temperature to $800{ }^{\circ} \mathrm{C}$ at a heating rate of $15{ }^{\circ} \mathrm{C} \mathrm{min}^{-1}$.

FT-IR spectra of pyrrole adsorbed on the catalyst samples were recorded on a Thermo Fisher Nicolet50 spectrometer, equipped with a MCT detector. Before placed into a quartz IR in situ cell equipped with $\mathrm{BaF}_{2}$ windows, the sample was firstly compressed into a self-supporting wafer (10-30 mg) and suffered from a heat treatment in $\mathrm{H}_{2}$ flow at $400{ }^{\circ} \mathrm{C}$ for $2 \mathrm{~h}$. 
Subsequently, the wafer was evacuated under vacuum for $3 \mathrm{~h}$ and then cooled to room temperature in He flow. After exposure to pyrrole vapor for $30 \mathrm{~min}$ and further evacuated for $1 \mathrm{~h}$, the IR spectra were recorded.

The XPS analyses were performed under vacuum $\left(>10^{-7} \mathrm{~Pa}\right)$ on Thermo Scientific ESCALAB 250Xi spectrometer with an Al $\mathrm{K} \alpha \mathrm{X}$-ray resource $(h \nu=1486.6 \mathrm{eV})$ after treatment with hydrogen at $400{ }^{\circ} \mathrm{C}$ in the reaction chamber. The spectral regions corresponding to $\mathrm{O} 1 \mathrm{~s}, \mathrm{Si} 2 \mathrm{p}$, Ti 2p, Ni 2p and $\mathrm{C} 1$ s core levels were recorded for each sample and referenced to $\mathrm{C} 1 \mathrm{~s}$ electron bond energy corresponding to graphitic carbon at $284.6 \mathrm{eV}$.

For solid-state ${ }^{29} \mathrm{Si}$ MAS NMR spectroscopy on Bruker Advance $600 \mathrm{MHz}$ spectrometer, samples were sealed in $4 \mathrm{~mm}$ OD rotors after $\mathrm{H}_{2}$ thermal treatment. A single-pulse sequence with ${ }^{1} \mathrm{H}$ TPPM decoupling was used to acquire ${ }^{29} \mathrm{Si}$ NMR spectra with a $59.67 \mathrm{MHz}$ operating resonance, a $\pi / 4$ pulse width (2.0 $\mu \mathrm{s})$ and a $30 \mathrm{~s}$ recycle delay. Each spectrum was obtained at ambient temperature after 1200 scans and the chemical shifts were referenced to tetramethylsilane $\left(\delta_{\text {iso }}=0 \mathrm{ppm}\right)$ by setting the isotropic peak of an external solid hexamethyldisiloxane (HMDS) sample to $6.7 \mathrm{ppm}$.

\subsection{Glycerol transesterification}

The synthesis of glycerol carbonate from transesterification of glycerol over series of ETS-10 solid base was performed in a $150 \mathrm{ml}$ three-neck round bottomed flask equipped with reflux condenser, magnetic stirrer and thermocouple. The heating energy was supplied constantly from oil bath and the temperature accuracy in the glass flask was monitored by an extra thermometer. Typically, glycerol was mixed with DMC in a certain proportion and heated to the desired temperature. Then, the transition metal loaded catalyst that has been reduced by hydrogen (flow rate: $150 \mathrm{~mL} \min ^{-1}$; purity: $\geq 99.999 \%$ ) at $400{ }^{\circ} \mathrm{C}$ for $4 \mathrm{~h}$ was added to the biphasic system, followed with stirring at specified rate. The formation of GC can be monitored roughly by system colour, and the transesterification process ends up with the presence of sole phase under assigned condition. After the reaction, the liquid reaction mixture was separated from the catalyst before analysis, and the remained catalyst was washed with methanol several times before drying and calcination for recycle utilization.

\subsection{Product analysis}

Qualitative and quantitative analysis of the liquid products were conducted with a gas chromatography equipped with a flame ionization detector and a DB-FFAP capillary column $(30 \mathrm{~m} \times$ $0.25 \mathrm{~mm} \times 0.25 \mu \mathrm{m})$. A $0.2 \mu \mathrm{L}$ injection was employed and nitrogen was used as the carrier gas. The FID was set to $270{ }^{\circ} \mathrm{C}$ and the inlet was isothermally maintained at $250{ }^{\circ} \mathrm{C}$ with a split ratio of $1: 50$. The oven parameters were programmed to start by increasing the temperature from $35{ }^{\circ} \mathrm{C}$ to $60{ }^{\circ} \mathrm{C}$ at a ramp of $10{ }^{\circ} \mathrm{C} \mathrm{min}$ min $^{-1}$ and was held at $60{ }^{\circ} \mathrm{C}$ for $1 \mathrm{~min}$. Then, it continuously went up to $230{ }^{\circ} \mathrm{C}$ at a rate of $15{ }^{\circ} \mathrm{C} \mathrm{min}$ min- $^{-1}$ and maintained at this temperature for $10 \mathrm{~min}$. The conversion of glycerol $\left(C_{\mathrm{GL}}\right)$ and the yield of $\mathrm{GC}\left(Y_{\mathrm{GC}}\right)$ were obtained based on the internal standard method with $n$-butanol as internal standard and calculated according to the following equations:

$$
\begin{gathered}
C_{\mathrm{GL}}=\frac{m_{\mathrm{GL}, \text { initial }}-m_{\mathrm{GL}, \text { residual }}}{m_{\mathrm{GL}, \text { initial }}} \times 100 \% \\
Y_{\mathrm{GC}}=\frac{m_{\mathrm{GC}}}{m_{\mathrm{GL}, \text { initial }}} \times 100 \%
\end{gathered}
$$

where $m_{\mathrm{GL} \text {,initial }}$ and $m_{\mathrm{GL}, \text { residual }}$ are the initial and residual mass weight of glycerol in the reaction system, respectively, and $m_{\mathrm{GC}}$ is the mass weight of obtained glycerol carbonate.

\section{Result and discussion}

\subsection{Catalysts characterization}

Fig. 1 displays the XRD patterns and $\mathrm{N}_{2}$ adsorption/desorption isotherms with pore size distributions inset. Obviously, when compared to ETS-10, METS-10 gives a lower XRD peak intensity, suggesting the negative impact of introducing mesopores on crystallinity. Likewise, series of transition metals play roles in decreasing material crystallinity to varying extent. Combining this result with $\mathrm{N}_{2}$ adsorption/desorption analysis data, introduction of transition metal into METS-10 seems to have significant influence on its BET-specific surface area, which decreases from 325.6 and $314.3 \mathrm{~m}^{2} \mathrm{~g}^{-1}$ of ETS-10 and METS-10, respectively, to the lowest $271.2 \mathrm{~m}^{2} \mathrm{~g}^{-1}$ of Zn/METS-10 (Table 1).

The reduced BET-specific surface area further demonstrates that the framework integrity of ETS-10 has been threatened to different degree by those transition metals, while pore size distributions are surprisingly in good keep, which all center around $16 \mathrm{~nm}$ and indicate not only their mesoporosity but also the absence of severe pore blockage by metal incorporation. And it is worth noting that both the BET-specific surface area $\left(298.5 \mathrm{~m}^{2} \mathrm{~g}^{-1}\right)$ and mesopore volume $\left(0.17 \mathrm{~cm}^{3} \mathrm{~g}^{-1}\right)$ of Ni/METS10 are apparently higher than other metallic samples, which matches well with the XRD result showing a superior intensity of characteristic peaks for Ni/METS-10. ICP results gives the metal contents in METS-10, from which can be seen that Fe and $\mathrm{Cu}$ suffers seriously lose during the catalyst preparation process. While for Ni-, Co-, Mn- and $\mathrm{Zn}$-supported samples, the measurable metal contents are close to theoretical values, which can be related to their chemical activity and tendency to form coordination compounds that strengthen their interaction with METS-10. Besides, due to the specific cation exchange capacity and accessibility for guest molecules of ETS-10 zeolite, metal species are allowed to take up its exchange sites during the impregnation process, and interact strongly with the zeolite support. Better yet, the lowered molar ratio of Si/Ti (3.2) from 5.5 further supports this conjecture.

Fig. 2 reveals the $\mathrm{CO}_{2}$-TPD results of ETS-10 and METS-10based samples, and the observed $\mathrm{CO}_{2}$ desorption temperatures are displayed in Table 2. As can be seen, except for the distinct peak around $140{ }^{\circ} \mathrm{C}$ due to the interaction between the weak basic surface hydroxyl groups in ETS-10 and $\mathrm{CO}_{2}$, there are also several peaks with rather weak intensity assigned to different strength of basicity. While, for METS-10, even though the total $\mathrm{CO}_{2}$ adsorption capacity has not been largely changed, 

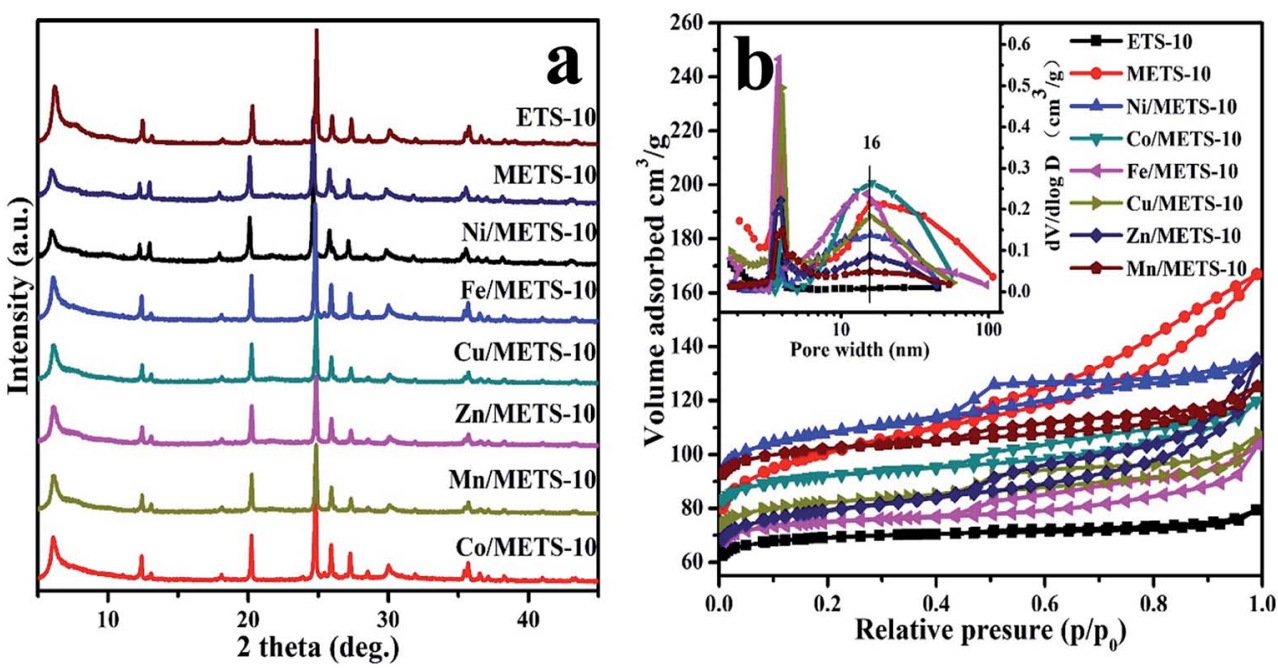

Fig. 1 (a) XRD patterns and (b) $\mathrm{N}_{2}$ adsorption/desorption isotherms and pore size distributions (inset, calculated using desorption branch) of transition metal loaded METS-10 samples (the patterns and isotherms of transition metal loaded samples have been offset to different degree along the vertical axis for clarity, respectively).

Table 1 Textural parameters of the catalyst samples

\begin{tabular}{|c|c|c|c|c|c|c|c|}
\hline Samples & Metal content ${ }^{a}(\mathrm{wt} \%)$ & $S_{\mathrm{BET}}^{b}\left(\mathrm{~m}^{2} \mathrm{~g}^{-1}\right)$ & $S_{\text {micro }}^{c}\left(\mathrm{~m}^{2} \mathrm{~g}^{-1}\right)$ & $S_{\text {meso }}^{d}\left(\mathrm{~m}^{2} \mathrm{~g}^{-1}\right)$ & $V_{\text {micro }}{ }^{e}\left(\mathrm{~cm}^{3} \mathrm{~g}^{-1}\right)$ & $V_{\text {meso }} f\left(\mathrm{~cm}^{3} \mathrm{~g}^{-1}\right)$ & Pore $(\mathrm{nm})$ \\
\hline ETS-10 & & 325.6 & 304.2 & 21.4 & 0.16 & 0.01 & 4 \\
\hline $\mathrm{M}_{1}$ ETS-10 & & 337.1 & 291.1 & 46.0 & 0.11 & 0.12 & 5 \\
\hline $\mathrm{M}_{3}$ ETS-10 & & 340.8 & 281.6 & 59.2 & 0.12 & 0.12 & 7 \\
\hline METS-10 & & 314.3 & 220.4 & 93.9 & 0.13 & 0.15 & 16 \\
\hline Ni/METS-10 & 4.9 & 298.5 & 204.8 & 93.7 & 0.13 & 0.17 & 16 \\
\hline $\mathrm{Cu} / \mathrm{METS}-10$ & 3.5 & 273.7 & 202.9 & 70.8 & 0.12 & 0.10 & 16 \\
\hline Zn/METS-10 & 4.4 & 271.2 & 204.8 & 66.4 & 0.07 & 0.11 & 16 \\
\hline Mn/METS-10 & 4.8 & 280.6 & 212.8 & 67.8 & 0.06 & 0.10 & 16 \\
\hline
\end{tabular}

${ }^{a}$ Determined by ICP. ${ }^{b}$ BET surface area. ${ }^{c}$ Microporous surface area. ${ }^{d}$ External surface area, obtained from $t$-plot method. ${ }^{e}$ Microporous pore volume, obtained from $t$-plot method. ${ }^{f}$ Mesoporous pore volume, obtained from BJH adsorption cumulative volume of pores between 1.7 and $300 \mathrm{~nm}$ in diameter.

the base strength distribution shifts drastically to high temperature that is attributed to the strong Lewis basic sites of $\mathrm{O}^{2-}$ anion, indicating the presence of more high-strength basic sites in METS-10 than that in ETS-10. When it comes to those metallic samples, their $\mathrm{CO}_{2}$ adsorption decreased unexpectedly, and Ni/METS-10, in particularly, presents mainly mediumstrength basic sites with the highest $\mathrm{CO}_{2}$ adsorption capacity, which is against previously reported conclusion that the basicity of cation-exchanged ETS-10 is directly related to the cation size. ${ }^{36,38}$ In fact, herein, basicity depends on not only the nature and composition of the transition metal but also the structure and pore topology of the zeolite. As a result, the cation size is definitely not the only driving factor, and it is worth making a delving into Ni/METS-10 for its distinctiveness.

Taking the unusual behaviour of Ni/METS-10 on $\mathrm{CO}_{2}$-TPD test into consideration, the basic property was further investigated by pyrrole-IR methods. For both METS-10 and Ni/METS10 , after treated at $400{ }^{\circ} \mathrm{C}$ in the $\mathrm{H}_{2}$ flow and vacuumed, the broad band around $3600 \mathrm{~cm}^{-1}$ disappeared, illustrating the absence of interaction between $\mathrm{OH}$ groups and water molecules. And seven other bands were detected in the high frequency region $\left(2700-3700 \mathrm{~cm}^{-1}\right)$ (Fig. 3a). The coexistence of two strong and broad bands around $3200-3400 \mathrm{~cm}^{-1}$ is taken as the main feature of $\mathrm{NH}$-stretching vibration resulting from the interaction of pyrrole with the Lewis basic sites varied in strength. ${ }^{39-41}$ Hence, in addition to the band at $3464 \mathrm{~cm}^{-1}$, there is also a broad band appearing at $3345 \mathrm{~cm}^{-1}$.

Because of the composition of ETS-10 zeolite including two kinds of alkaline cations $\left(\mathrm{Na}^{+}\right.$and $\left.\mathrm{K}^{+}\right)$, the appearance of this band confirms the adsorption of pyrrole on framework oxygen adjacent to $\mathrm{Na}^{+} / \mathrm{K}^{+}$. Besides, when compared to that for METS10, this band becomes weak after Ni species impregnated, which can be explained as the cation exchange has taken place to a large extent between $\mathrm{Na}^{+} / \mathrm{K}^{+}$and $\mathrm{Ni}^{2+}$ in METS-10 framework. The assignment of the bands around 3127 and $3104 \mathrm{~cm}^{-1}$ for the four $\mathrm{CH}$ stretching modes (every band used twice) of 


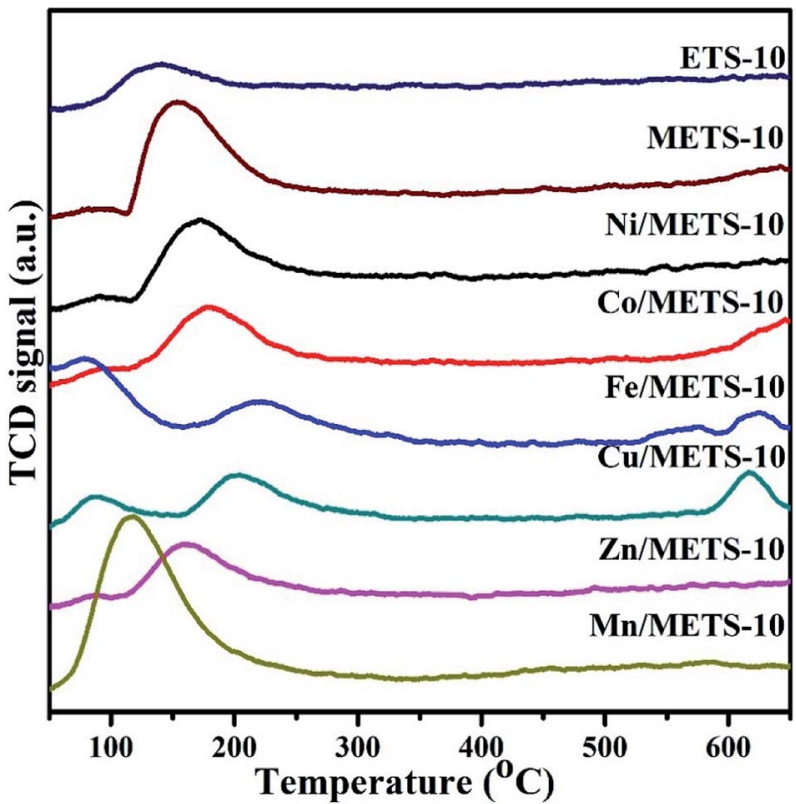

Fig. $2 \mathrm{CO}_{2}$-TPD profiles of ETS-10, METS-10 and series of transition metals loaded METS-10 samples.

liquid pyrrole can also be accepted here, but slight blueshift of the two bands occurred. The band at $3054 \mathrm{~cm}^{-1}$ close to the $\mathrm{CH}$ bands is reported to be derived from the removal of the band degeneration after pyrrole adsorption. ${ }^{42,43}$ The two combination bands at 2940 and $2850 \mathrm{~cm}^{-1}$ powerfully demonstrate the existence of strong basic sites. Fig. 3b shows the IR spectra in the region of $1250-1700 \mathrm{~cm}^{-1}$ for samples evacuated at $65^{\circ} \mathrm{C}$ to investigate the associated pyrrole ring-stretching modes. Since the physically adsorbed pyrrole can be removed by evacuation process, it is believed that the band around $1470 \mathrm{~cm}^{-1}$ can only result from the presence of Lewis basic sites. What's more, to our gratification, no Lewis/Brønsted acid sites can be found in the samples with the absence of the band around $1490 \mathrm{~cm}^{-1}$, indicating the distinct Lewis basicity of METS-10 and Ni/METS10.

As shown in Fig. 4, the SEM images of both METS-10 and Ni/ METS-10 exhibit the characteristic morphology with particle size of $18-23 \mu \mathrm{m}$ (Fig. $4 \mathrm{a}$ and b), and it is delightful that $\mathrm{Ni}$ species can interact with METS-10 not so aggressively that keeps crystal appearance relatively intact. The TEM images of the thinsectioned METS-10 and Ni/METS-10 samples directly evidence

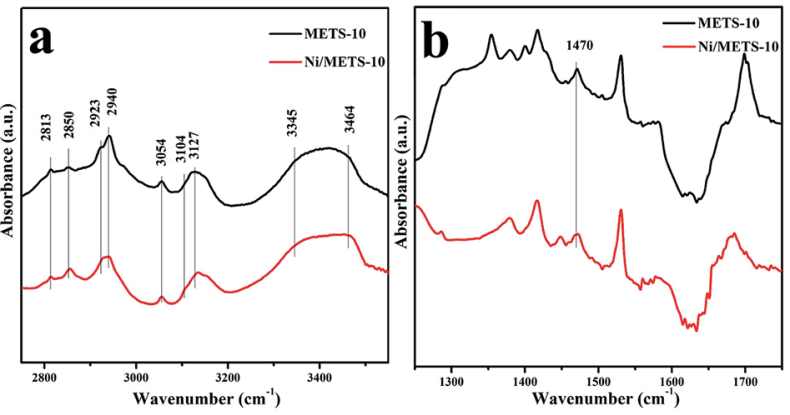

Fig. 3 Pyrrole-IR spectra of METS-10 and Ni/METS-10 (a) in the high frequency region $\left(2700-3700 \mathrm{~cm}^{-1}\right)$ and $(b)$ in the ring-stretching region $\left(1250-1700 \mathrm{~cm}^{-1}\right)$.

the existence of abundant and well dispersed hierarchical mesopores (Fig. 4c and d). What's more, the size of these mesopores is highly uniform and its range is consistent with that shown by the $\mathrm{N}_{2}$ sorption (Fig. 1b). Notably, though ICP analysis shows an $4.9 \mathrm{wt} \%$ content of $\mathrm{Ni}$ in the Ni/METS-10 sample, there are no obvious Ni phases observed in the XRD patterns, SEM-EDS analysis and TEM images, indicating that the Ni species could be highly dispersed in Ni/METS- 10 .

In order to go into the surface composition and electronic state details of active sites on the Ni-based catalyst, XPS experiment of the in situ reduced catalyst was performed. The Ni $2 \mathrm{p}$ XPS spectrum of the catalyst is shown in Fig. 5 with six kinds of characteristic peaks being observed. The binding energy (BE) of two weak peaks at $\sim 853$ and $\sim 870 \mathrm{eV}$ can be attributed to $\mathrm{Ni}^{0}$ $2 \mathrm{p}_{3 / 2}$ and $2 \mathrm{p}_{1 / 2}$ phases, ${ }^{44-46}$ respectively. And the peaks appearing at 856.8 and $874.4 \mathrm{eV}$ are $\sim 2.0 \mathrm{eV}$ higher than those reported binding energies corresponding to NiO, which can be assigned to the presence of $\mathrm{Ni}^{2+}$ species at the zeolite exchange sites. Herein, combining these shifts with catalyst support function, it can be inferred that there should be a significant metal-support interaction. In this way, the ionic nickel(II) species presenting in the form as $\mathrm{NiO}$ and nickel titanates/silicates can be strongly stabilized at the outer surface and the large cavities of the zeolite. Except the main peaks mentioned above, the Ni 2p XPS spectrum also exhibits two surrounding shake-up satellite peaks centered at 862.7 and $880.6 \mathrm{eV}$, which further explains the location of $\mathrm{Ni}^{2+}$ species at zeolite exchange sites. On the whole, XPS results indicate that there are two different Ni species in the Ni/METS-10 after reduction at $673 \mathrm{~K}$, and the nickel atomic concentration calculated from their corresponding peaks intensity is $6.8 \%$ with a mass content of $11.9 \mathrm{wt} \%$.

Table 2 Basic properties of the catalysts samples

\begin{tabular}{|c|c|c|c|c|c|c|c|c|c|}
\hline$\frac{\text { Samples }}{\text { Ni/METS-10 }}$ & \multicolumn{8}{|c|}{ Temperature of peak $\left({ }^{\circ} \mathrm{C}\right)$} & $\frac{\mathrm{CO}_{2} \text { adsorption }\left(\mathrm{cm}^{3} \mathrm{~g}^{-1} \mathrm{STP}\right)}{2.32}$ \\
\hline Co/METS-10 & 90 & 180 & 265 & 375 & 470 & 510 & - & 650 & 2.07 \\
\hline $\mathrm{Cu} / \mathrm{METS}-10$ & 90 & 205 & 315 & 440 & 477 & 540 & 570 & 615 & 2.07 \\
\hline Zn/METS-10 & 90 & 160 & 285 & 426 & 490 & 588 & 628 & 680 & 2.07 \\
\hline Mn/METS-10 & - & 115 & 300 & 458 & 528 & 588 & 635 & 654 & 2.01 \\
\hline
\end{tabular}



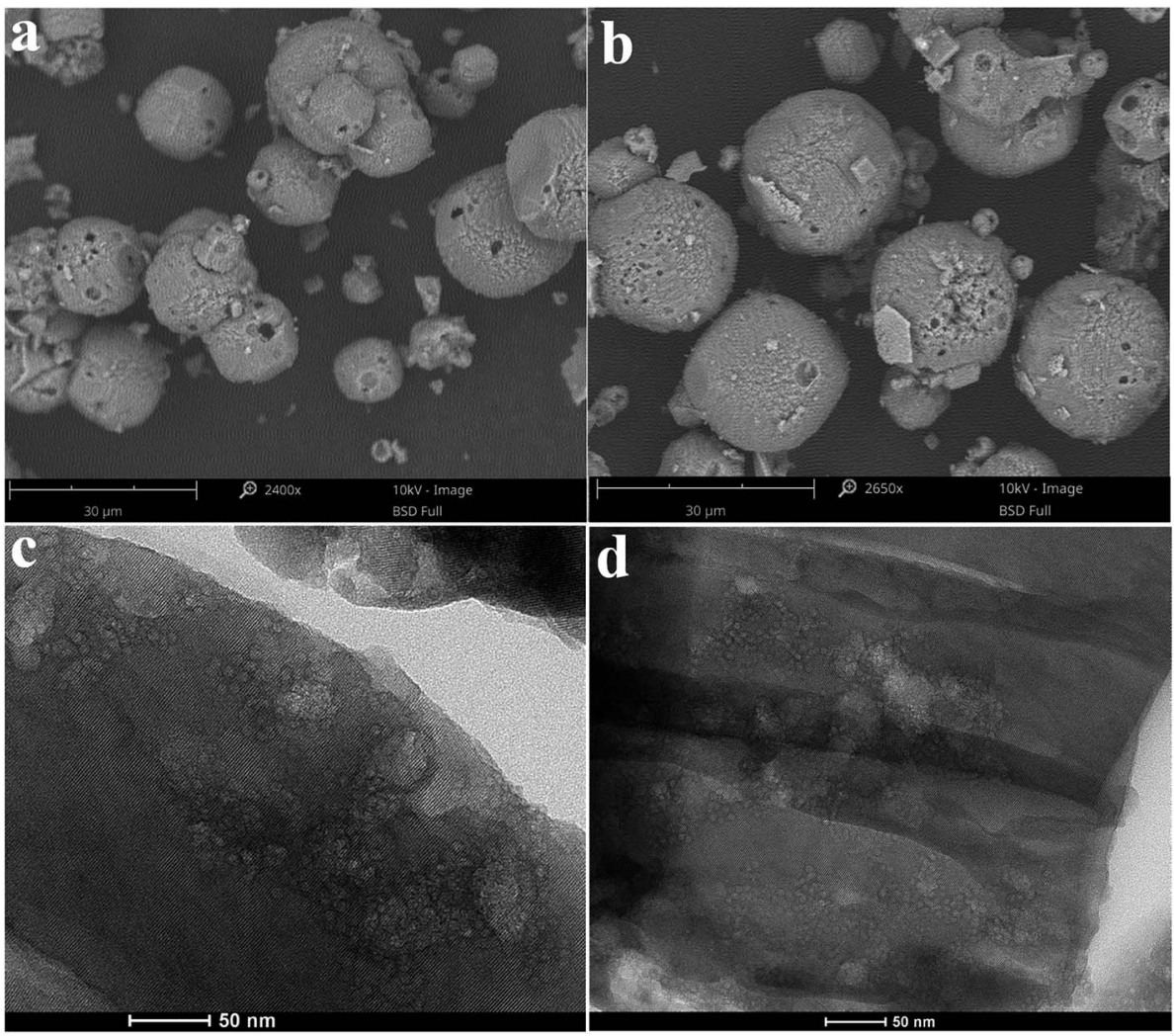

Fig. 4 (a) and (b) SEM images of METS-10 and Ni/METS-10 samples, (c) and (d) TEM images (magnification power: 86000 $\times$ ) of the thinsectioned METS-10 and Ni/METS-10 sample (the light dots are mesopores).

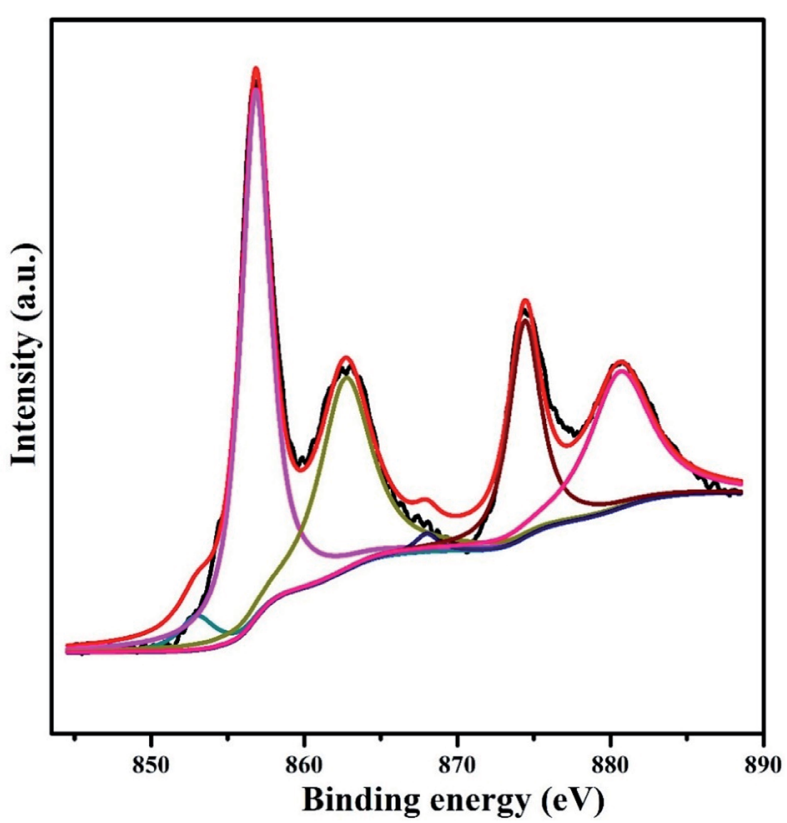

Fig. 5 XPS spectrum of $\mathrm{Ni} 2 \mathrm{p}$ for Ni/METS-10 sample.

The Ti $2 \mathrm{p}_{3 / 2}$ peak of the ETS-10 samples positioned around $459 \mathrm{eV}$ (Fig. 6a) is related to the octahedral Ti species in the -O-Ti-O-Ti-O- chains, while at lower BE ( $\sim 457.7 \mathrm{eV})$, a small peak was also observed for both METS-10 and Ni/METS-10, which is due to the existence of titanium species with different states from the octahedral ones such as those extraframework $\mathrm{TiO}_{2} \cdot{ }^{47}$ Besides, although the two samples share nearly the same location of Ti 2p spectra, the full widths at half maximum (FWHMs) appear evident inconsistent. Compared Ni/METS-10 with METS-10, it is obvious that the principal peak width for Ti $2 \mathrm{p}$ is broader, reinforcing the presence of multistates titanium species and defects in zeolites. What's more, the broaden peak width can also come from the perpendicular $\mathrm{TiO}_{6}$ octahedral linear chains being isolated and distributed uniformly in the siliceous matrixes. The O1s binding energy region of Ni/METS-10 and METS-10 is shown in Fig. $6 \mathrm{~b}$ with three types of $\mathrm{O} 1 \mathrm{~s}$ peaks observed. Among them, the two peaks at slightly higher BE of 532.4 and $531 \mathrm{eV}$ are attributed to $\mathrm{O}$ in $\mathrm{Si}-\mathrm{O}-\mathrm{Si}$ and $\mathrm{Si}-\mathrm{O}-\mathrm{Ti}$ linkages, while the last one at low $\mathrm{BE}$ is owing to Ti-O-Ti bonds. For Ni/METS-10, even though the BE of $\mathrm{O} 1 \mathrm{~s}$ peak was almost maintained, the peak intensity was decreased especially for those at higher BE. This is probably due to the removal of silicon atoms, which then result in exposure of $\mathrm{Ti}$ on the surface.

Fig. 7 shows the ${ }^{29} \mathrm{Si}$ MAS NMR spectrum for Ni/METS-10 with two characteristic sharp peaks around $-96.7 \mathrm{ppm}$ and $-107 \mathrm{ppm}$ assigned to $\mathrm{Si}(3 \mathrm{Si}, 1 \mathrm{Ti})$ and $\mathrm{Si}(4 \mathrm{Si}, 0 \mathrm{Ti})$, respectively. ${ }^{48}$ Typically, there are two types of silicon chemical environments derived from five different crystallographic Si sites, among which the $\mathrm{Si}\left[(\mathrm{OSi})_{3}(\mathrm{OTi})_{1}\right]$ site especially exhibits four resonance peaks in the region of -94 to $-97 \mathrm{ppm}^{.9-51}$ 

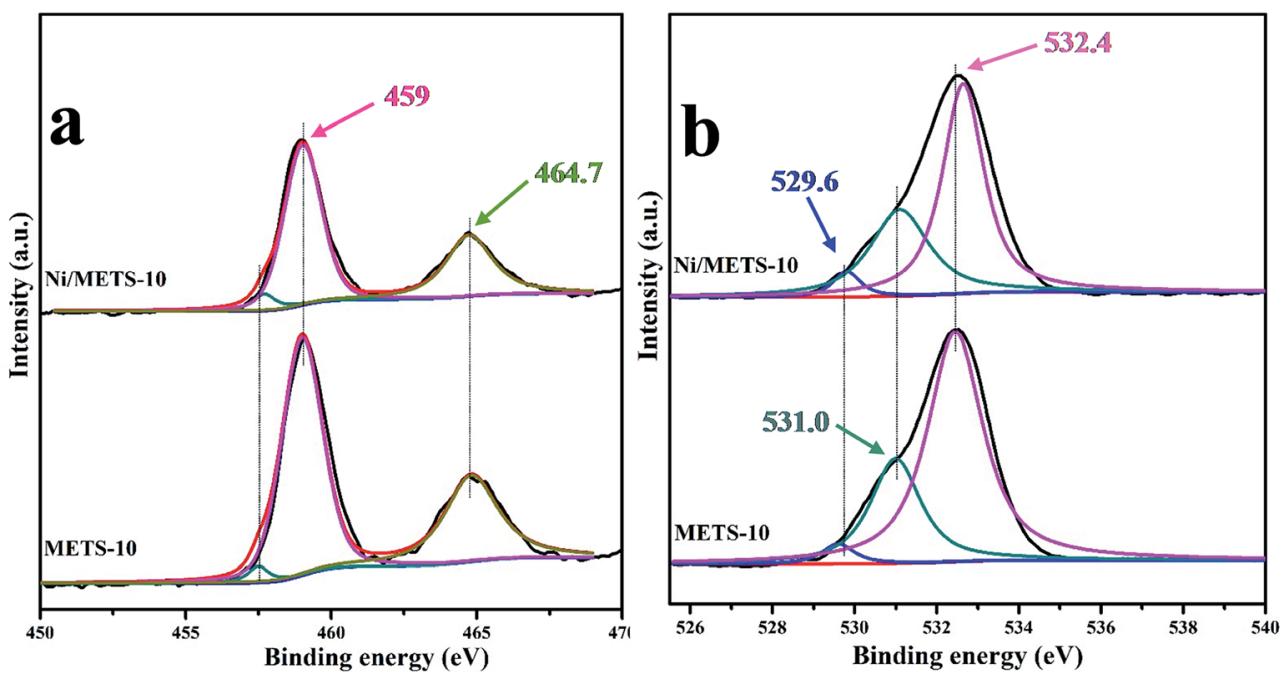

Fig. 6 XPS spectra of (a) the Ti 2p region and (b) O 1s region for both Ni/METS-10 and METS-10 samples.

Obviously, compared with previous work, Ni/METS-10 gives rather different resonance pattern with only two peaks observed, indicating the discernible distortion of silicon sites and the presence of defects. This could be due to the incorporation of $\mathrm{Ni}$ species that are substituted for $\mathrm{Si}$ atoms at (3Si) sites and the introduction of hierarchical pores into ETS-10 framework.

$\mathrm{H}_{2}$-TPR was employed to further study the behaviour of surface active sites (Ni species) and their interaction with zeolite support (Fig. 8). As can be seen, the reduction begins at $303{ }^{\circ} \mathrm{C}$ and there are three apparent peaks centred around 555, 646 and $700{ }^{\circ} \mathrm{C}$ consisting of Ni/METS-10 TPR profile, suggesting that the $\mathrm{Ni}^{2+}$ species have been reduced in different support positions. The most salient peak at the highest temperature with

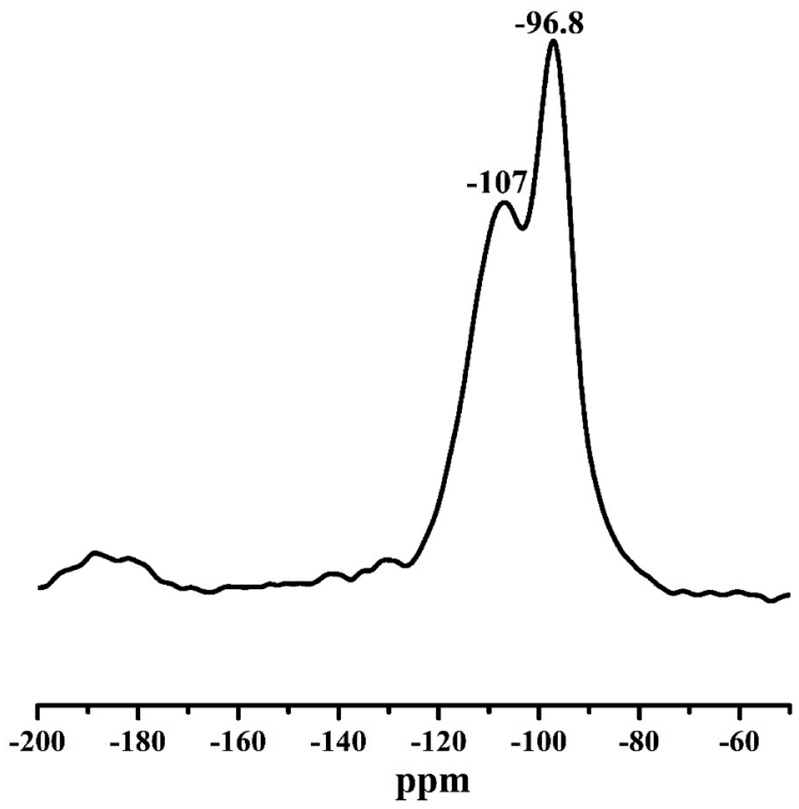

Fig. $7{ }^{29}$ Si NMR MAS spectrum of Ni/METS-10 sample. a shoulder peak at a little bit lower temperature is possible rooted from nickel titanates/silicates detected in XPS spectra, such as $\mathrm{NiSiO}_{3}$, indicating the cationic $\mathrm{Ni}$ located on $\left(\mathrm{TiO}_{6}\right)^{2-}$ octahedral $/ \mathrm{SiO}_{4}$ tetrahedral and their strong interaction with METS-10 zeolite. Those smaller and broader peaks at relatively lower temperature are very likely derived from the reduction of $\mathrm{Ni}_{2} \mathrm{O}_{3}$ to the metallic $\mathrm{Ni}$, which matches well with the XPS results and more importantly, exhibits a low reducibility of the Ni/METS-10 catalyst. To evaluate the dispersion of $\mathrm{Ni}$ species, the reduced Ni/METS-10 was further studied by CO pulse chemisorption after acquiring the definite proportions of $\mathrm{Ni}$ species by EDS technique. According to the CO chemisorption, the active particle diameter and the metallic surface area that are generally believed to be the essential factors in conducting catalyst reactivity is $9.6 \mathrm{~nm}$ and $29.5 \mathrm{~m}^{2} \mathrm{~g}^{-1}$, respectively. The

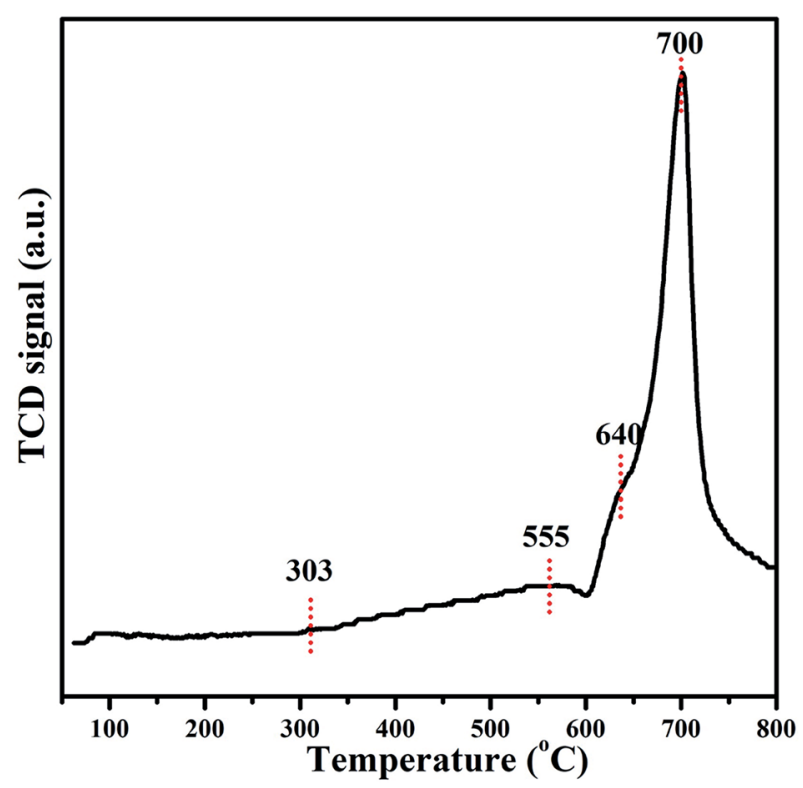

Fig. $8 \mathrm{H}_{2}$-TPR profile of Ni/METS-10 sample. 

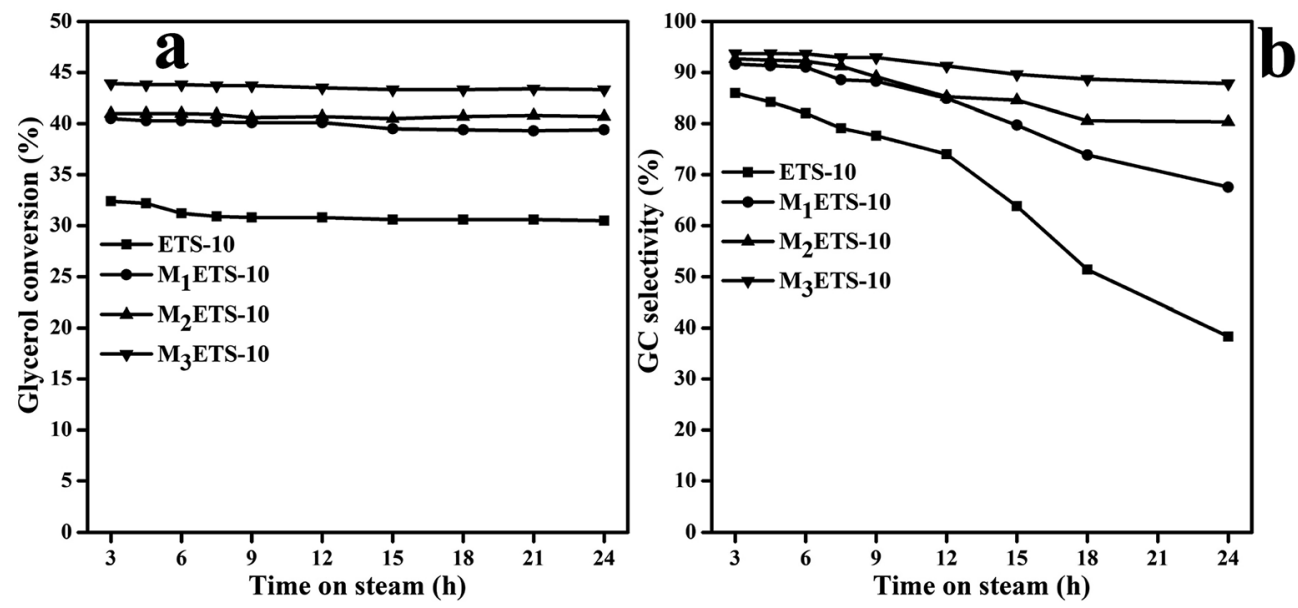

Fig. 9 Glycerol conversion and GC selectivity on different catalysts (reaction conditions: $2.5 \mathrm{~g}$ glycerol, $5 \mathrm{~g} \mathrm{DMC}, 0.1 \mathrm{~g} \mathrm{catalyst,} 90^{\circ} \mathrm{C}$ ).

dispersion of nickel species that can be influenced by their reduction degree and was calculated from the two parameters above is $20.6 \%$.

\subsection{Effect of hierarchical porous structure on glycerol transesterification}

It is well known that both the acidity/basicity and porosity of zeolite influence greatly their catalytic performance. The former has been widely reported that plays critical role in the intrinsic activity and selectivity of the catalyst, while the presence of hierarchical pores mainly devotes to promoting the accessibility of active sites by improving the transportation of guest molecules and suppressing the coke formation. ${ }^{52,53}$ As a result, series of catalysts sharing with comparable basicity (the $\mathrm{CO}_{2}$ adsorption all around $2 \mathrm{~cm}^{3} \mathrm{~g}^{-1}$ ) but obviously different hierarchical porous topologies were adopted to explore the relationship between the hierarchical structure and the catalytic performance. Fig. 9a and b present the glycerol conversion and GC selectivity variations with the time-on-stream (TOS). It can be seen from Fig. 9a that all the selected catalysts show their highest catalytic activity at the beginning $3 \mathrm{~h}$ of the reaction and then keep the glycerol conversion stable at a certain level after a marginal decrease. However, the conventional ETS-10 obviously gives the lowest glycerol conversion and suffers the most evident and rapidest drop of product selectivity from $86.0 \%$ to $36.3 \%$. On the contrary, the other three catalysts $\left(\mathrm{M}_{1} \mathrm{ETS}-10\right.$, $\mathrm{M}_{2}$ ETS-10 and $\mathrm{M}_{3}$ ETS-10) with porous structure all deactivated much slower than ETS-10, and their stabilities not surprisingly follow the same order with their pore size and external surface area. The $\mathrm{M}_{3}$ ETS-10 with the widest pore size distribution of $7 \mathrm{~nm}$, largest surface area of $340.8 \mathrm{~m}^{2} \mathrm{~g}^{-1}$ and external surface area of $59.2 \mathrm{~m}^{2} \mathrm{~g}^{-1}$ displays the best performance and can keep the catalytic activity stable as long as $24 \mathrm{~h}$. For $\mathrm{M}_{2}$ ETS-10 and $\mathrm{M}_{1}$ ETS-10, though in the worse case when compared with $M_{3}$ ETS-10, they still perform better than ETS-10 with enhanced glycerol conversion and GC selectivity. In fact, based on the Kelvin equation, there can be seriously steric hindrance in the zeolite micropores for those reactions composed by reactants with certain pore size, which can selectively condense in the mesopores and external surface regions. ${ }^{53-55}$ Generally, the introduction of hierarchical pores into zeolite catalysts is beneficial to the improvement of catalytic activity and lifetime. According to Fig. 9b, the advantage of yielding desired product on hierarchical ETS-10 zeolites is apparent and stands time, which might be attributed to the high molecular transport rate of the hierarchical pores that facilitate the effective absorption of reactants on active sites and thus reduce the occurrences of side reactions.

\subsection{Optimisation of transesterification reaction conditions}

3.3.1 Effect of impregnated metals. It has been discussed above that series of catalysts mentioned in present work are endowed with different chemical properties by incorporation of various metals, especially their related basicity that is widely deemed to be of vital importance for glycerol transesterification reaction. However, in the present work, for $\mathrm{Ni}, \mathrm{Co}, \mathrm{Zn}, \mathrm{Cu}, \mathrm{Fe}$ and Mn wet-impregnated METS-10 catalysts, their catalytic activity in transesterification reaction varies in the order $\mathrm{Ni} /$ METS-10 > Zn/METS-10 > Mn/METS-10 > Fe/METS-10 > Co/ METS-10 $>\mathrm{Cu} /$ METS-10, which is clearly affected by more than basicity. Ni/METS-10 predictably performed the best in converting glycerol to GC with the strongest basicity strength. However, when it comes to $\mathrm{Zn}, \mathrm{Fe}$, $\mathrm{Co}$ and $\mathrm{Cu}$ impregnated METS-10 catalysts, though they share similar $\mathrm{CO}_{2}$ adsorption quantity, their reactivity still jagged obviously. As shown in Fig. 10a, both glycerol conversion and GC yield of Zn/METS-10 ( $91 \%$ and $89.4 \%$, respectively) approximate to that of $\mathrm{Ni} /$ METS-10 (97.1\% and 91.6\%, respectively) and are unexpectedly much higher than that of other metal catalysts. Likewise, $\mathrm{Mn} / \mathrm{ETS}-10$ is not competitive at all in basicity, but it seems more active than $\mathrm{Co}, \mathrm{Fe}$ and $\mathrm{Cu}$-incorporated samples. $\mathrm{Co} /$ METS-10 and Cu/METS-10 prove to be the least effective ones with the transesterification conversion and product yield all below $60 \%$. These unusual phenomenon could be resulted from the finally detected quantity and dispersion of active phase and the damage to zeolite structure by introduction of different 

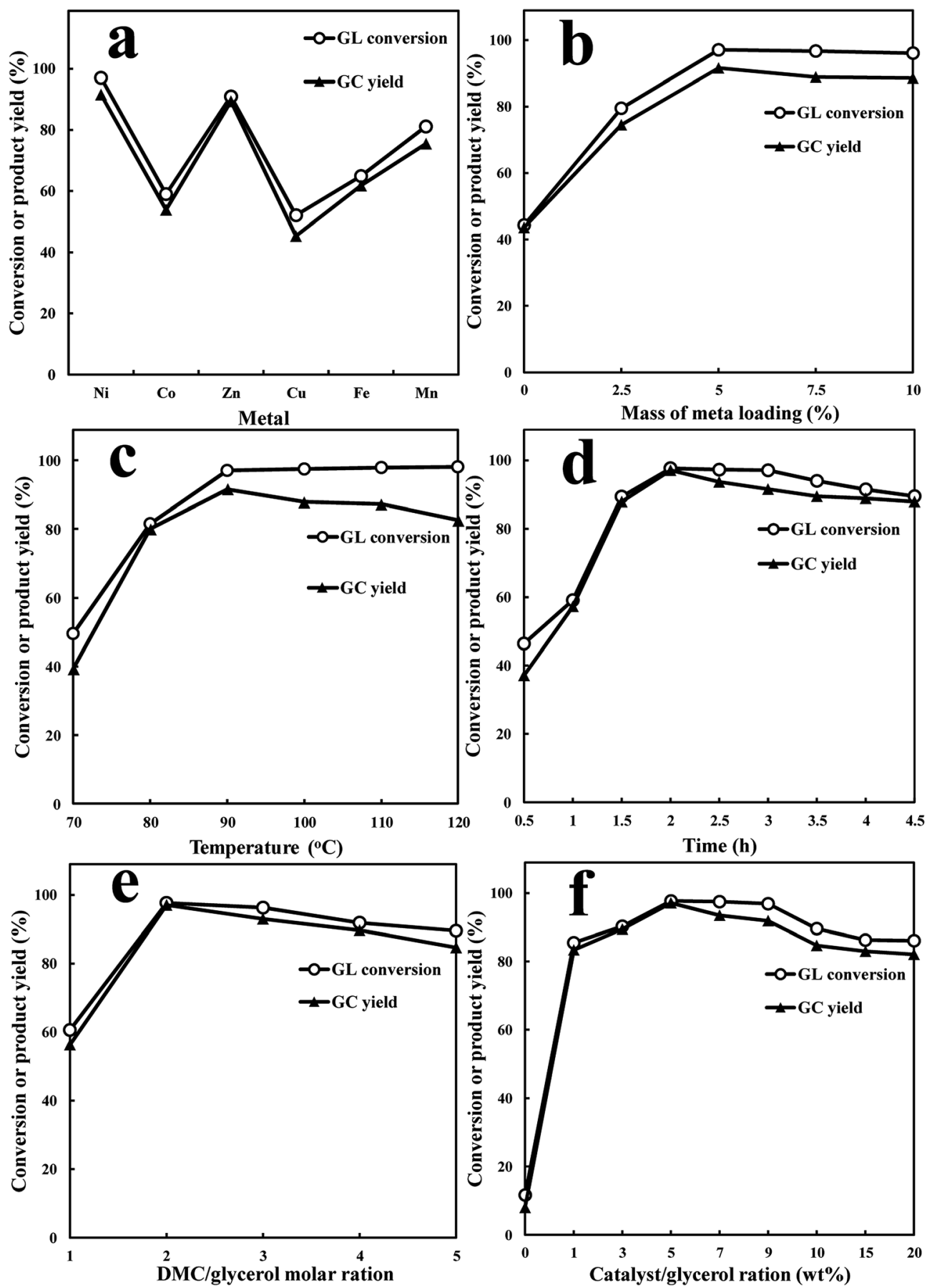

Fig. 10 Optimization of reaction conditions for glycerol transesterification on Ni/MEST-10.

metals with various degrees. It is worth mentioning that without metal impregnation and structural damages the performance of METS-10 and ETS-10 follows the general order of their basic strength. On the basics of the $\mathrm{CO}_{2}$-TPD results, except for the presence of weak-strength basic sites that is similar to ETS-10, there are also appreciable quantity of medium- and highstrength basic sites in METS-10 zeolite, which seems to make the main contributions to the increased catalytic efficiency. Furthermore, when compared to those metal catalysts, METS-10 and ETS-10 show evidently lower catalytic activity, indicating the requirement and significance of metal participating for facilitating the reaction. As a result, the overall GC yield decreases in the order Ni/METS-10 (91.6\%) > Zn/METS-10 $(89.4 \%)>$ Mn/METS-10 (75.5\%) > Fe/METS-10 (61.8\%) > Co/ MET S-10 (53.9\%) > Cu/METS-10 (45.3\%) > METS-10 (43.6\%) > ETS-10 (39.2\%). Thus, this confirms the conjecture that the promoting effect of introducing transition metal for glycerol conversion can be closely related to not only the inherent properties and framework structure of support but also the interaction of metal-support that can derive various influence 


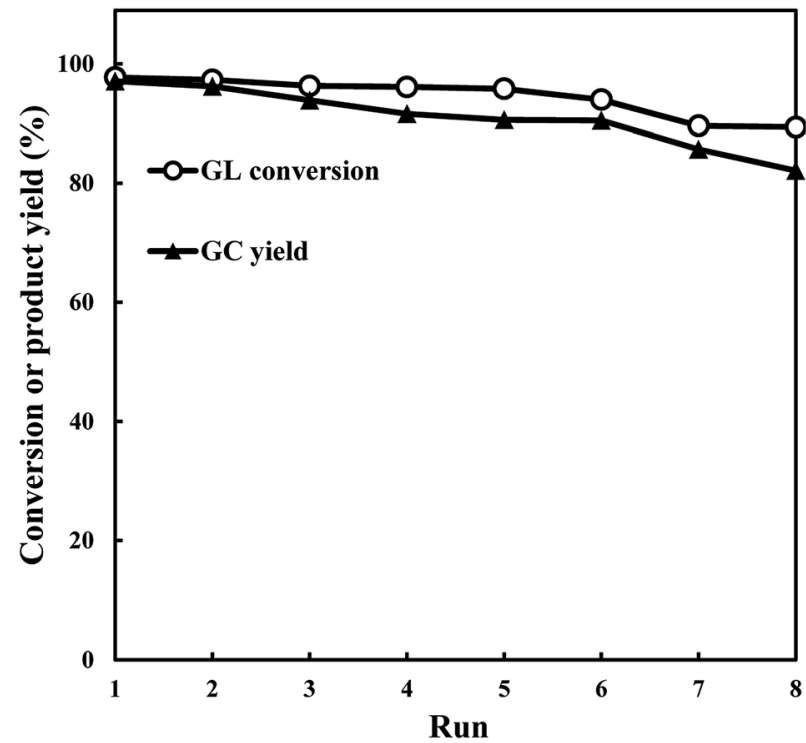

Fig. 11 The reusability of the catalyst in transesterification reaction over Ni/METS-10 under optimized conditions (reaction conditions: $2.5 \mathrm{~g}$ glycerol, $5 \mathrm{~g} \mathrm{DMC}, 0.125 \mathrm{~g} \mathrm{Ni} / \mathrm{METS}-10,90^{\circ} \mathrm{C}, 2 \mathrm{~h}$ ).

factors including actual metal content, dispersion and existing state.

3.3.2 Effect of metal loadings. An appropriate metal loading is of vital importance for metal dispersion and then play a crucial role in reaction activity. Therefore, based on the comparison among series of metal catalysts for glycerol transesterification above, Ni/MEST-10 is chosen in this section to explore the optimal metal content required here. It can be seen from Fig. 10b that increasing metal loading can effectively improve catalytic activity and the maximum GC yield (91.6\%) can be obtained by participation of $5 \mathrm{wt} \% \mathrm{Ni}$. It seems that continuous increasing the metal loading may not necessarily motivate the reaction, which led to $1.7 \%$ and $3 \%$ lower GC yield at $7.5 \mathrm{wt} \%$ and $10 \mathrm{wt} \%$ of $\mathrm{Ni}$, respectively. This can be attributed to that though low Ni loadings benefit its dispersion that is also possible to be further decreased by the low reduction degree caused by the strong interaction of $\mathrm{Ni}$ species and zeolite, it cannot offer enough surface active sites. In contrast, the surface active sites can be increased by higher Ni loadings. However, excessive $\mathrm{Ni}$ contents inevitably encourage the serious aggregation of Ni particles and lead to structural damage of zeolite, thus go against the glycerol transesterification reaction.

3.3.3 Effect of reaction temperature. The influence of reaction temperature on the conversion and product distribution was explored and shown in Fig. 10c. It can be found that an increase in the reaction temperature from $70{ }^{\circ} \mathrm{C}$ to $120{ }^{\circ} \mathrm{C}$ results in an overall improvement of the conversion of glycerol from $49.6 \%$ to $98.1 \%$ and of the yield towards GC from $39.2 \%$ to $82.6 \%$, suggesting the positive role of higher temperature in promoting the transesterification reaction. However, the optimal condition is at $90{ }^{\circ} \mathrm{C}$ that is the boiling temperature of DMC yielding $91.6 \%$ GC. After that, the reaction activity declined but maintained at a steady level. This corresponds to Arrhenius equation that increasing reaction temperature can improve the miscibility of the multiphase reaction system and collision rate between the reactants, and thus facilitate the reaction rate, which could benefit product yield.

3.3.4 Effect of reaction time. Reactions were also performed as a function of time over Ni/HMETS-10. As presented in Fig. 10d, both the glycerol conversion and GC yield are maximized when the reaction time is extended to $2 \mathrm{~h}$, with $97.7 \%$ glycerol conversion and $97.1 \%$ GC yield obtained. It has been reported previously that for glycerol, the reactivity of both the first and second $\mathrm{OH}$ group is faster than the third one, which requires more reaction time to make sure the full access of these $\mathrm{OH}$ groups to DMC molecules and facilitate the formation of the thermodynamically stable cyclic carbonate..$^{56}$ Consequently, here in this work, longer reaction time allows the biphasic system of glycerol and DMC to mix well and to contact with active sites more thoroughly, thus, leading to a more complete transesterification reaction. Whereas, further prolonging the time to $4.5 \mathrm{~h}$ seems to do nothing positive for catalytic performance but keeps the GC yield above $85 \%$ all the time, suggesting that reaction time does not takes a pivotal position in
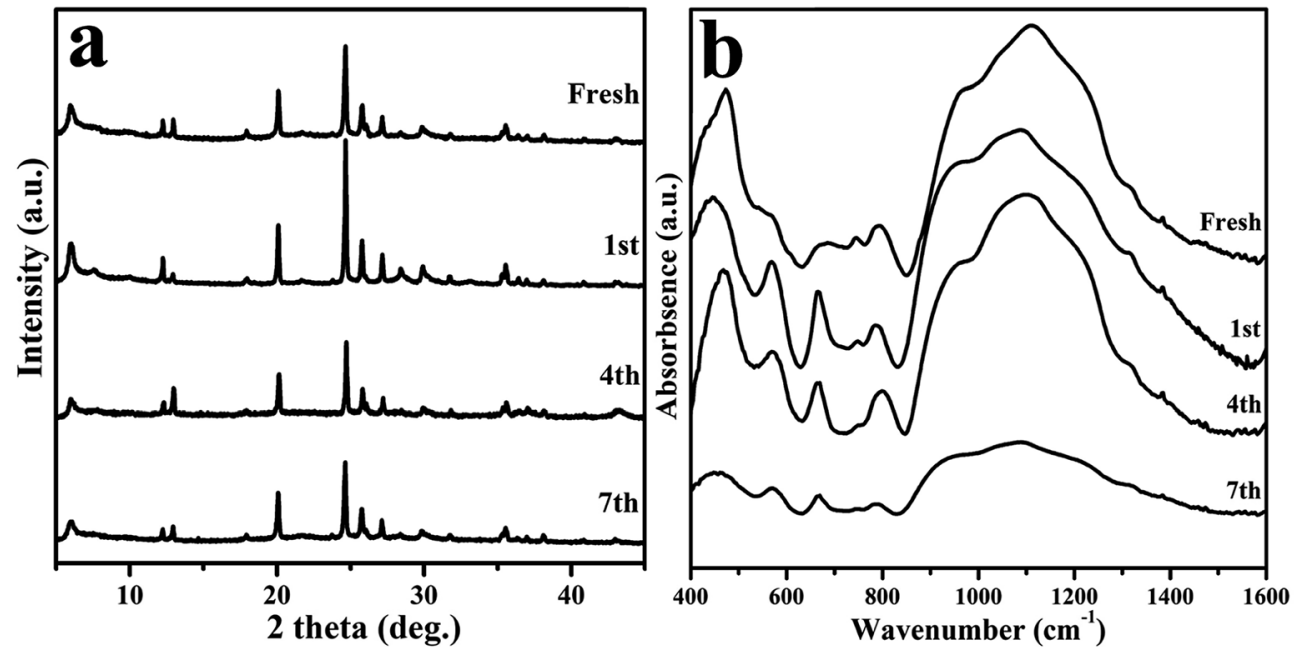

Fig. 12 (a) XRD patterns, (b) FT-TR spectra of fresh and used Ni/METS-10 samples. 


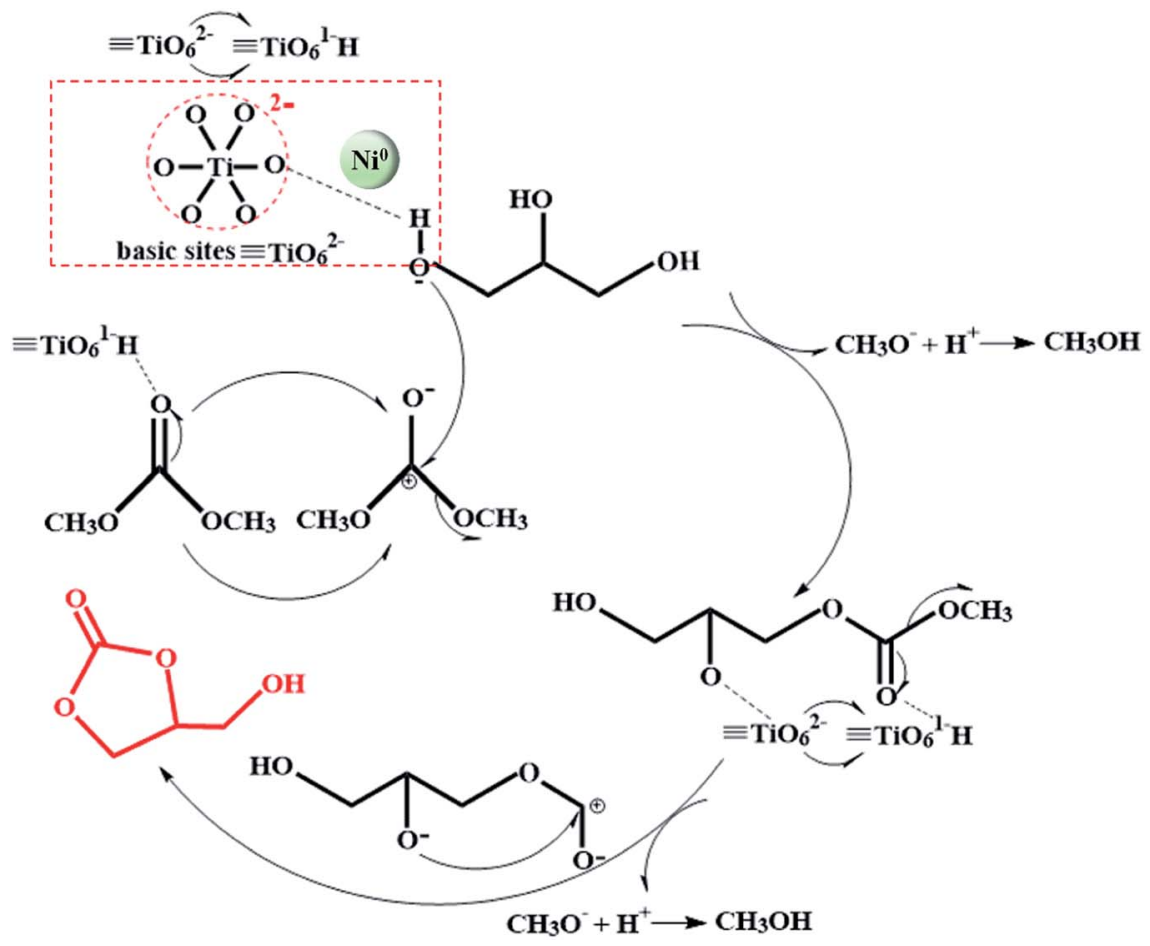

Scheme 1 Possible reaction pathway for the glycerol transesterification reaction.

yielding GC in a wide range of 1.5-4.5 h. Besides, the unapparent product concentration change also illustrates an approximate equilibrium for the reaction. Herein, it is better to operate the catalytic system for $2 \mathrm{~h}$ from a comprehensive consideration.

3.3.5 Effect of DMC/glycerol molar ratio. Since the concentration of substrates is important and can be used to effectively manipulate the reaction rate, the impact that various ratios of DMC to glycerol play on GC yield is investigated in this work. According to the stoichiometric calculations, the molar ratio of $\mathrm{DMC} /$ glycerol required during the transesterification process is exactly $1: 1$, but as a matter of fact, considering the biphasic system resulted from the hydrophilic glycerol and hydrophobic DMC, excessive amount of DMC is always applied to increase the contact between glycerol and DMC, drive forward the reaction equilibrium and prevent the reversibility of the reaction. Besides, given the influence of different catalysts and reaction conditions that bring diverse interaction of glycerol and active sites, the DMC/glycerol ratio often varies in a wide range. Herein, from Fig. 10e it can be seen that when the reaction was performed at $90{ }^{\circ} \mathrm{C}$ for $2 \mathrm{~h}$, both the optimal glycerol conversion and GC yield can be preserved by increasing the DMC/glycerol molar ratio to $2: 1$. After that, the reactivity gradually decreased with the DMC contents further increased, which can be owing to the counterproductive behaviour from overmany DMC that not only diluting the reaction but also encouraging the occurrence of possible side reactions. Moreover, adding too many DMC does not stand the economic point of view. As a result, the molar ratio of DMC/glycerol is better to be fixed at $2: 1$ in the following experiments.
3.3.6 Effect of catalyst/glycerol mass ratio. The amount of catalyst is considered to be closely related to the active sites indispensable for remarkably catalytic performance. And in general, more addition of catalyst means that more active sites is available for reactants adsorption. Fig. 10f shows an expected tendency of GC yield change, which climbs up to peak value of $97.1 \%$ at a $5 \mathrm{wt} \%$ mass ratio of catalyst/glycerol. Then, it fell below $90 \%$ with the catalyst/glycerol mass ratio further increased to $10 \mathrm{wt} \%$, and it continues downwards to $82.1 \%$ finally at the mass ratio of $20 \mathrm{wt} \%$. This could be due to that being access to more and more necessary active sites greatly accelerates the glycerolysis rate and facilitates the corresponding intermediate formation, thus, benefits the combination of the original two immiscible reactants and leads to the formation of GC. While, though the presence of too many basic sites is beneficial to promoting the reaction rate, it foster the side reaction at the same time that leads to the formation of undesired products and in turn reduction of GC yield. What's more, an excess of catalyst loading also possibly causes mass transfer limitation from the reactants to the catalytic active sites and particle agglomeration, which goes against yielding GC. Hence, a $5 \mathrm{wt} \%$ catalyst/glycerol mass ratio is adopted in the present work to satisfy the active sites need for glycerol transesterification.

\subsection{Stability and reusability of catalyst}

Taking the significance of separating the product and reusing the catalyst for industrial applications into account, recycling experiments were carried out to test the stability and reusability 
of catalyst. The catalyst was separated by filtration after each reaction, and then dried at $100{ }^{\circ} \mathrm{C}$ overnight, followed by calcination at $475{ }^{\circ} \mathrm{C}$ in air for $4 \mathrm{~h}$ to remove the deposited carbon. The catalytic reaction was tested again with the reduced catalyst. At least 8 experimental runs were performed and the results are shown in Fig. 11. It seems that both the glycerol and GC yield did not suffer much from the experimental runs with just slight drop to $89.4 \%$ and $82.4 \%$, respectively, suggesting the retention of excellent catalytic performance. The XRD, FT-IR, and inductively coupled plasma (ICP) experiments were carried out to explore the influence of recycling test on the catalyst. As shown in Fig. 12a, the XRD peak intensity of the used catalyst is obviously lower than that of the fresh catalyst, while both catalysts share similar FT-IR spectra (Fig. 12b), which could be interpreted as the formation of surface carbon deposition and occurrence of small structural changes but not serious enough to destroy the framework architectures of catalyst completely. Furthermore, according to the ICP analysis, a certain amount of Ni species leaching $(0.9,1.6$ and $1.6 \%$ for the first, fourth and seventh runs, respectively) should be mainly responsible for the decreased catalytic performance. Thus, it is desirable in the future study to focus on stabilization of the catalyst through immobilization of active metal species.

\subsection{Mechanism investigation}

The synthesis process of GC by glycerol transesterification over reduced Ni/METS-10 was investigated according to the base catalytic principle and the synergistic effects of active metal (Scheme 1). Notably, the catalyst used in this study must be reduced before experiments, otherwise, its catalytic activity is almost as low as those catalysts without metal species introduced. In other words, it emphasizes the critical role of $\mathrm{Ni}^{0}$ species. Based on the results of $\mathrm{H}_{2}$-TPR and XPS, there is a certain amount of elemental nickel after catalyst reduction, which has been widely reported being greatly competitive and potential for hydrogenation/hydrogenolysis process. It is deduced that the presence of $\mathrm{Ni}^{0}$ species here could accelerate the interaction of Lewis basic sites $\left(\mathrm{TiO}_{6}{ }^{2-}\right)$ with active glycerol hydroxyl groups, and thus facilitate the deprotonation of the more reactive primary hydroxyl group to form the glyceroxide anion. In this course, $\mathrm{Ni}^{0}$ species appear to be a pulling force driving the acidic hydrogen proton on primary alcohol to tend towards $\mathrm{TiO}_{6}{ }^{2-}$. Meanwhile, the carbonyl carbon of DMC is activated by $\mathrm{TiO}_{6}{ }^{1-} \mathrm{H}$ and attacked by the oxygen of primary alcohol, leading to the elimination of methoxy group on DMC in the form of methanol. And then, it will continuously suffer attack of the oxygen from the secondary alcohol, with which the formed unstable intermediate species will be fast transformed into the desired product GC by intramolecular cyclisation process. Similarly, another methanol molecular is produced during this step.

Given the declined conversion and GC yield that can be resulted from raising reaction temperature, prolonging reaction time, excessive addition of DMC and catalyst, it is possible to incur the side reaction in taking place. For example, further carbonylation and decarboxylation under the action of superabundant basic sites will result in the formation of undesired products glycerol dicarbonate (GDC) and glycidol (GD).

\section{Conclusions}

In conclusion, series of ETS-10 zeolite catalysts were used to investigate the detailed process of GC formation from glycerol transesterification. For all the tested catalysts, the introduction of hierarchically porous structure into zeolite ETS-10 presented obvious advantage in exposing more basic sites, and avoiding the porous blockage and structural damage by metal incorporation as much as possible. Furthermore, among different transition metals (Fe, Co, Ni, $\mathrm{Cu}, \mathrm{Zn}$, and $\mathrm{Mn}$ ), Ni was found to be the most suitable for being loaded on METS-10, which led to an enhanced catalyst basicity without serious loss of zeolite crystallinity. More importantly, $\mathrm{Ni}^{0}$ species from the necessary operation of catalyst reduction were turned out to be vital for accelerating the access of reactants to the Lewis basic sites $\left(\mathrm{TiO}_{6}{ }^{2-}\right)$. As a result, the reduced Ni/METS-10 with the exact metal content, the best metal dispersion, the relatively integrated catalyst structure and the strongest Lewis basic strength proved to be the most reactive in catalytic conversion glycerol transesterification, resulting in a $97.7 \%$ glycerol conversion and 97.1\% GC yield under mild condition. The last but not least, $\mathrm{Ni}$ / METS-10 can maintain the catalytic performance after being recycled at least 8 times, indicating its excellent stability and reusability.

\section{Author contributions}

The manuscript was written through contributions of all authors. All authors have given approval to the final version of the manuscript.

\section{Conflicts of interest}

The authors declare no conflict of interest.

\section{Acknowledgements}

This work was supported by the National Natural Science Foundation of China under Grant No. 21878048 and 21676055.

\section{References}

$1 \mathrm{~J}$. R. Ochoa-Gómez, O. Gómez-Jiménez-Aberasturi, C. A. Ramírez-López, J. Nieto-Mestre, B. Maestro-Madurga and M. Belsué, Synthesis of glycerol carbonate from 3chloro-1,2-propanediol and carbon dioxide using triethylamine as both solvent and $\mathrm{CO}_{2}$ fixation-activation agent, Chem. Eng. J., 2011, 175, 505-511.

2 C. A. G. Quispe, C. J. R. Coronado and J. A. Carvalho, Glycerol: production, consumption, prices, characterization and new trends in combustion, Renewable Sustainable Energy Rev., 2013, 27, 475-493. 
3 G. T. Ang, K. T. Tan and K. T. Lee, Recent development and economic analysis of glycerol-free processes via supercritical fluid transesterification for biodiesel production, Renewable Sustainable Energy Rev., 2014, 31, 61-70.

4 J. L. Hu, J. J. Li, Y. L. Gu, Z. H. Guan, W. L. Mo, Y. M. Ni, T. Li and G. X. Li, Oxidative carbonylation of glycerol to glycerol carbonate catalyzed by $\mathrm{PdCl}_{2}(\mathrm{phen}) / \mathrm{KI}, A p p l$. Catal., A, 2010, 386, 188-193.

5 M. J. Climent, A. Corma, P. D. Frutos, S. Iborra, M. Noy, A. Velty and P. Concepcion, Chemicals from biomass: synthesis of glycerol carbonate by transesterification and carbonylation with urea with hydrotalcite catalysts, $J$. Catal., 2010, 269, 140-149.

6 Y. T. Algoufi, U. G. Akpan, M. Asif and B. H. Hameed, Onepot synthesis of glycidol from glycerol and dimethyl carbonate over KF/sepolite catalyst, Appl. Catal., A, 2014, 487, 181-188.

7 M. Aresta, A. Dibenedetto, F. Nocito and C. Ferragina, Valorization of bio-glycerol: new catalytic materials for the synthesis of glycerol carbonate via glycerolysis of urea, $J$. Catal., 2009, 268, 106-114.

8 M. O. Sonnati, S. Amigoni, T. Darmanin and O. Choulet, Glycerol carbonate as a versatile building block for tomorrow: synthesis, reactivity, properties and applications, Green Chem., 2013, 15, 283-306.

9 J. S. Choi, F. S. H. Simanjuntaka, J. Y. Oh, K. I. Lee, S. D. Lee, M. Cheong, H. S. Kim and H. Lee, Ionic-liquid-catalyzed decarboxylation of glycerol carbonate to glycidol, J. Catal., 2013, 297, 248-255.

10 M. Aresta, A. Dibenedetto, F. Nocito and C. A. Pastore, Study on the carboxylation of glycerol to glycerol carbonate with carbon dioxide: the role of the catalyst, solvent and reaction conditions, J. Mol. Catal. A: Chem., 2006, 257, 149153.

11 A. Dibenedetto, A. Angelini, M. Aresta, J. Ethiraj, C. Fragale and F. Nocito, Converting wastes into added value products: from glycerol to glycerol carbonate, glycidol and epichlorohydrin using environ-mentally friendly synthetic routes, Tetrahedron, 2011, 67, 1308-1313.

12 J. R. Ochoa-Gómez, O. Gómez-Jiménez-Aberasturi, C. Ramírez-López, J. Nieto-Mestre, B. Maestro-Madurga and M. Belsué, Synthesis of glycerol carbonate from 3chloro-1,2-propanediol and carbon dioxide using triethylamine as both solvent and $\mathrm{CO}_{2}$ fixation-activation agent, Chem. Eng. J., 2011, 175, 505-511.

$13 \mathrm{~J} . \quad$ R. Ochoa-Gómez, O. Gómez-Jiménez-Aberasturi, C. Ramírez-López and M. Belsué, A brief review on industrial alternatives for the manufacturing of glycerol carbonate, a green chemical, Org. Process Res. Dev., 2012, 16, 389-399.

14 M. K. Munshi, S. M. Gade, M. V. Mane, D. Mishra, S. Pal and K. Vanka, Chemical in glycerol carbonate synthesis, J. Mol. Catal. A: Chem., 2014, 391, 144-149.

15 P. Tundo and M. Selva, The Chemistry of Dimethyl Carbonate, Acc. Chem. Res., 2002, 35, 706-716.
16 D. Wang, B. Yang, X. Zhai and L. Zhou, Synthesis of diethyl carbonate by catalytic alcoholysis of urea, Fuel Process. Technol., 2007, 88, 807-812.

17 S. C. Kim, Y. H. Kim, H. Lee, D. Y. Yoon and B. K. Song, Lipase-catalyzed synthesis of glycerol carbonate from renewable glycerol and dimethyl carbonate through transesterification, J. Mol. Catal. B: Enzym., 2007, 49, 75-78.

$18 \mathrm{~J}$. R. Ochoa-Gómez, O. Gómez-Jiménez-Aberasturi, B. Maestro-Madurga, A. Pesquera-Rodríguez, C. RamírezLópez, L. Lorenzo-Ibarreta, J. Torrecilla-Soria and M. C. Villarán-Velasco, Synthesis of glycerol carbonate from glycerol and dimethyl carbonate by transesterification: catalyst screening and reaction optimization, Appl. Catal., A, 2009, 366, 315-324.

19 A. Takagaki, K. Iwatani, S. Nishimura and K. Ebitani, Synthesis of glycerol carbonate from glycerol and dialkyl carbonates using hydrotalcite as a reusable heterogeneous base catalyst, Green Chem., 2010, 12, 578-581.

20 M. G. Álvarez, M. Plíšková, A. M. Segarra, F. Medina and F. Figueras, Synthesis of glycerol carbonates by transesterification of glycerol in a continuous system using supported hydrotalcites as catalysts, Appl. Catal., B, 2012, 113, 212-220.

21 M. Gupta, Manufacturing processes for emulsifiers, Bailey's Industrial Oil \& Fat Products, John Wiley \& Sons, Inc., New York, 1996, vol. 4, pp. 569-601.

22 A. Takagaki, K. Iwatani, S. Nishimura and K. Ebitani, Synthesis of glycerol carbonate from glycerol and dialkyl carbonates using hydrotalcite as a reusable heterogeneous base catalyst, Green Chem., 2010, 12, 578-581.

23 A. Z. Abdullaha, T. Y. Wibowoa and R. Zakariaa, Effect of tetramethyl ammonium hydroxide on the activity of $\mathrm{LiOH}$ intercalated montmorillonite catalyst in the transesterification of methyl laurate with glycerol, Chem. Eng. J., 2011, 167, 328-334.

24 F. S. H. Simanjuntak, T. K. Kim, S. D. Lee, B. S. Ahn, H. S. Kim and H. Lee, CaO-catalyzed synthesis of glycerol carbonate from glycerol and dimethyl carbonate: Isolation and characterization of an active Ca species, Appl. Catal., A, 2011, 401, 220-225.

25 M. Du, Q. Li, W. Dong, T. Geng and Y. Jiang, Synthesis of glycerol carbonate from glycerol and dimethyl carbonate catalyzed by $\mathrm{K}_{2} \mathrm{CO}_{3} / \mathrm{MgO}$, Res. Chem. Intermed., 2012, 38, 1069-1077.

$26 \mathrm{P}$. Lu, H. Wang and K. Hu, Synthesis of glycerol carbonate from glycerol and dimethyl carbonate over the extruded CaO-based catalyst, Chem. Eng. J., 2013, 228, 147-154.

27 M. Malyaadri, K. Jagadeeswaraiah, P. S. Sai Prasad and N. Lingaiah, Synthesis of glycerol carbonate by transesterification of glycerol with dimethyl carbonate over Mg/Al/Zr catalysts, Appl. Catal., A, 2011, 401, 153-157.

28 R. X. Bai, S. Wang, F. M. Mei, T. Li and G. X. Li, Synthesis of glycerol carbonate from glycerol and dimethyl carbonate catalyzed by KF modified hydroxyapatite, J. Ind. Eng. Chem., 2011, 17, 777-781.

29 F. S. H. Simanjuntak, V. T. Widyaya, C. S. K., B. S. Ahn, Y. J. Kim and H. Lee, Synthesis of glycerol carbonate from 
glycerol and dimethyl carbonate using magnesiumlanthanum mixed oxide catalyst, Chem. Eng. Sci., 2013, 94, 265-270.

30 D. Singh, B. Reddy, A. Ganesh and S. Mahajani, Zinc/ Lanthanum Mixed-Oxide Catalyst for the Synthesis of Glycerol Carbonate by Transesterification of Glycerol, Ind. Eng. Chem. Res., 2014, 53, 18786-18795.

31 S. E. Kondawar, C. R. Patil and C. V. Rode, Tandem Synthesis of Glycidol via Transesterification of Glycerol with DMC over Ba-Mixed Metal Oxide Catalysts, ACS Sustainable Chem. Eng., 2017, 5, 1763-1774.

32 M. Xiang, X. J. Ni, X. F. Yi, A. M. Zheng, W. C. Wang, M. Y. He, J. Xiong, T. Y. Liu, Y. L. Ma, P. Y. Zhu, X. Zheng and T. D. Tang, Preparation of Mesoporous Zeolite ETS-10 Catalysts for High-Yield Synthesis of $\alpha, \beta$-Epoxy Ketones, ChemCatChem, 2015, 7, 521-525.

33 X. J. Ni, M. Xiang, W. Q. Fu, Y. L. Ma, P. Y. Zhu, W. C. Wang, M. Y. He, K. Q. Yang, J. Xiong and T. D. Tang, Direct synthesis of mesoporous zeolite ETS-10 and Ni-ETS-10 with good catalytic performance in the Knoevenagel reaction, $J$. Porous Mater., 2016, 2, 423-429.

34 M. Xiang, J. N. Liu, W. Q. Fu, T. D. Tang and D. F. Wu, Improved Activity for Cellulose Conversion to Levulinic Acid Through Hierarchization of ETS-10 Zeolite, ACS Sustainable Chem. Eng., 2017, 5, 5800-5809.

35 M. W. Anderson, O. Terasaki, T. Ohsuna, A. Philippou, S. P. MacKay, A. Ferreira, J. Rocha and S. Lidin, Structure of the microporous titanosilicate ETS-10, Nature, 1994, 367, 347-351.

36 P. Liu, M. Derchi and E. J. M. Hensen, Promotional effect of transition metal doping on the basicity and activity of calcined hydrotalcite catalysts for glycerol carbonate synthesis, Appl. Catal., B, 2014, 144, 135-143.

37 A. Philippou and M. W. Anderson, Aldol-Type Reactions Over Basic Microporous Titanosilicate ETS-10 Type Catalysts, $J$. Catal., 2000, 189, 395-400.

38 S. B. Waghmode, R. Vetrivel, S. G. Hegde, C. S. Gopinath and S. Sivasanker, Physicochemical Investigations of the Basicity of the Cation Exchanged ETS-10 Molecular Sieves, J. Phys. Chem. B, 2003, 107, 8517-8523.

39 P. Scokart and P. Rouxhet, Characterization of the Basicity of Oxides Through the Infrared Study of Pyrrole Adsorption, $J$. Chem. Soc., Faraday Trans., 1980, 76, 1476-1489.

40 M. M. Huang and S. Kaliaguin, Zeolite Basicity Characterized by Pyrrole Chemisorption: An Infrared Study, J. Chem. Soc., Faraday Trans., 1992, 88, 751-758.

41 M. Huang and S. Kaliaguine, Lewis acid and Lewis basic sites in alkali-exchanged zeolites characterization and catalytic activity, Stud. Surf. Sci. Catal., 1992, 73, 291-300.
42 D. Barthomeuf, Basic Zeolites: Characterization and Uses in Adsorption and Catalysis, Catal. Rev., 1996, 38, 521-612.

43 D. Barthomeuf and A. Mallmann, Basicity and Electronegativity of Zeolites, Innovation in Zeolite Materials Science, 1987, vol. 37, pp. 365-374.

44 S. Badrinarayanan, R. I. Hegde, I. Balakrishnan, S. B. Kulkarni and P. Ratnasamy, XPS study of nickel in NiHZSM5 catalysts, J. Catal., 1981, 71, 439-442.

45 S. Narayanan, XPS studies on the reduction of nickel mordenite, Zeolites, 1984, 4, 231-234.

46 J. K. Kesavan, I. Luisetto, S. Tuti, C. Meneghini, C. Battocchio and G. Iucci, Ni supported on YSZ: XAS and XPS characterization and catalytic activity for $\mathrm{CO}_{2}$ methanation, J. Mater. Sci., 2017, 52, 10331-10340.

47 L. Lv, F. Y. Lee, J. Zhou, F. Su and X. S. Zhao, XPS study on microporous titanosilicate ETS-10 upon acid treatment, Microporous Mesoporous Mater., 2006, 96, 270-275.

48 M. W. Anderson, J. Rocha, Z. Lin, A. Philippou, I. Orion and A. Ferreira, Isomorphous substitution in the microporous titanosilicate ETS-10, Microporous Mater., 1996, 6, 195-204.

49 J. Rocha, A. Ferreira, Z. Lin and M. W. Anderson, Synthesis of microporous titanosilicate ETS-10 from $\mathrm{TiCl}_{3}$ and $\mathrm{TiO}_{2}$ : a comprehensive study, Microporous Mesoporous Mater., 1998, 23, 253-263.

50 A. Eldewik and R. F. Howe, Cobalt substitution in ETS-10, Microporous Mesoporous Mater., 2001, 48, 65-71.

51 Y. Liu, F. Chen, R. E. Wasylishen, Z. H. Xu, J. Sawada and S. M. Kuznicki, A Study of Silver Species on SilverExchanged ETS-10 and Mordenite by XRD, SEM and SolidState ${ }^{109} \mathrm{Ag},{ }^{29} \mathrm{Si}$ and ${ }^{27} \mathrm{Al}$ NMR Spectroscopy, J. Nanosci. Nanotechnol., 2012, 12, 6420-6427.

52 L. H. Chen, X. Y. Li, J. C. Rooke, Y. H. Zhang, X. Y. Yang, Y. Tang, F. S. Xiao and B. L. Su, Hierarchically structured zeolites: synthesis, mass transport properties and applications, J. Mater. Chem., 2012, 22, 17381-17403.

53 H. B. Zhang, Z. J. Hu, L. Huang, H. X. Zhang, K. S. Song, L. Wang, Z. P. Shi, J. X. Ma, Y. Zhuang, W. Shen, Y. H. Zhang, H. L. Xu and Y. Tang, Dehydration of Glycerol to Acrolein over Hierarchical ZSM-5 Zeolites: Effects of Mesoporosity and Acidity, ACS Catal., 2015, 5, 2548-2558.

54 Y. T. Kim, K. D. Jung and E. D. Park, A comparative study for gas-phase dehydration of glycerol over H-zeolites, Appl. Catal., A, 2011, 393, 275-287.

55 P. A. Monson, Contact Angles, Pore Condensation, and Hysteresis: Insights From a Simple Molecular Model, Langmuir, 2008, 24, 12295-12302.

56 P. U. Okoye, A. Z. Abdullah and B. H. Hameed, Stabilized ladle furnace steel slag for glycerol carbonate synthesis via glycerol transesterification reaction with dimethyl carbonate, Energy Convers. Manage., 2017, 133, 477-485. 